\title{
Comorbidities and medication use in patients with a recent clinical fracture at the Fracture Liaison Service
}

\author{
L. Vranken ${ }^{1,2}$ (1) - C. E. Wyers ${ }^{1,2}$ - R. Y. Van der Velde ${ }^{1,2}$ - H. M. Janzing ${ }^{3}$. \\ S. Kaarsemaker ${ }^{4}$ P. P. Geusens ${ }^{5,6}$ - J. P. Van den Bergh ${ }^{1,2,6}$
}

Received: 26 June 2017 / Accepted: 25 October 2017 /Published online: 23 November 2017

(C) The Author(s) 2017. This article is an open access publication

\begin{abstract}
Summary In this cross-sectional study, two-thirds of Fracture Liaison Service (FLS) patients had comorbidities and medications associated with increased bone- or fall-related fracture risk. Bone-related and fall-related fracture risk (BRR and FRR) were associated with age and fracture type, but not with gender or BMD. Systematic evaluation of these factors leads to a more profound assessment in FLS care.

Introduction This study is a systematic evaluation of comorbidities and medications associated with increased fracture risk in patients aged 50-90 years with a recent fracture visiting the FLS.

Methods In this cross-sectional cohort study, comorbidities were classified according to ICD-10 and medications according to the Anatomic Therapeutic Chemical (ATC) classification and further categorized into those associated BRR and FRR.

Results Of 1282 patients ( $72 \%$ women; $65 \pm 9$ years), 53\% had at least one BRR, $46 \%$ had at least one FRR, and $66 \%$ at
\end{abstract}

Electronic supplementary material The online version of this article (https://doi.org/10.1007/s00198-017-4290-y) contains supplementary material, which is available to authorized users.

L. Vranken

1.vranken@maastrichtuniversity.nl

Department of Internal Medicine, VieCuri Medical Centre, P.O. Box 1926, 5900 BX Venlo, The Netherlands

2 Department of Internal Medicine, NUTRIM School of Nutrition and Translational Research in Metabolism, Maastricht University Medical Centre+ (Maastricht UMC+), P.O. Box 616, 6200 MD Maastricht, The Netherlands least one BRR and/or FRR. At least one BRR, as well as at least one FRR were associated with age, BMI, and fracture type, but not with gender or BMD. The proportion of patients with only BRR $( \pm 20 \%)$ or only FRR $( \pm 10 \%)$ was similar among ages, gender, BMI, fracture type, and BMD. The combination of at least one BRR and at least one FRR was significantly associated with age, BMI, and major fractures, but not with gender or BMD.

Conclusion Comorbidities and medications associated with increased fracture risk are present in two-thirds of patients visiting the FLS. In addition, the proportion of patients having a combination of BRR and FRR increased significantly with age, BMI, and fracture severity. This indicates that systematic evaluation of these factors is important for a more profound assessment of subsequent fracture risk in FLS care.

Keywords Fracture prevention $\cdot$ Fracture risk assessment . Osteoporosis
3 Department of Surgery, VieCuri Medical Centre, P.O. Box 1926, 5900 BX Venlo, The Netherlands

4 Department of Orthopaedic Surgery, VieCuri Medical Centre, P.O. Box 1926, 5900 BX Venlo, The Netherlands

5 Department of Internal Medicine, Subdivision Rheumatology, CAPHRI, Maastricht University Medical Centre+ (Maastricht UMC+), P.O. Box 616, 6200 MD Maastricht, The Netherlands

6 Biomedical Research Centre, Hasselt University, Agoralaan, Gebouw D, 3590 Diepenbeek, Belgium 


\section{Introduction}

Fractures constitute a major health concern, as the lifetime risk of a clinical fracture at the age of 50 years is $50 \%$ for women and $20 \%$ for men $[1,2]$. The annual number of fractures is expected to increase due to aging of the population [3]. It is well documented that prior fractures in adulthood increase the risk of future fractures [4-6]. Prior fractures are associated with an approximately 2 -fold increased relative risk (RR) for subsequent fractures [6]. Furthermore, the subsequent fracture risk is highest immediately after the fracture [7]. Hence, a fracture is an opportunity to prevent future fractures. Therefore, in current osteoporosis guidelines [8-12], secondary fracture prevention is recommended in all patients aged 50 years or older with a recent clinical fracture. The Fracture Liaison Service (FLS) has been identified as the most successful approach for secondary fracture prevention [11-13].

Risk factors contributing to fracture are numerous and include factors with a deleterious effect on bone and that increase fall risk or both. Some of these risk factors are potentially modifiable. There are no studies that systematically evaluated all comorbidities and medication with an increased fracture risk in patients with a recent fracture. Systematic evaluation of comorbidities and medications could contribute to specify and quantify the presence of bone- and fall-related (BRR and FRR) risk factors for fractures. In this study, we systematically evaluated comorbidities and medications with an increased fracture risk in patients aged 50-90 years with a recent clinical fracture visiting the FLS.

\section{Materials and methods}

\section{Study design and population}

A cross-sectional cohort study was conducted among women and men with a recent clinical vertebral or non-vertebral fracture who were evaluated at the FLS of the VieCuri Medical Centre located in The Netherlands. Identified were all consecutive patients aged 50-90 years with a recent clinical fracture visiting the emergency department from January 2009 until June 2011. All fractures were radiologically confirmed. After fracture repair, a specialized nurse screened all patients and invited those eligible for fracture risk evaluation to the FLS. Patients with facial/skull and finger/toe fractures, metastatic cancer in bone, fracture due to high-energy trauma, osteomyelitis, or failure of prosthesis were excluded. Those willing and able to be evaluated visited the FLS approximately 3 to 4 months after the fracture event. According to the Dutch guideline for treatment of osteoporosis [8], patients received a detailed questionnaire for evaluation of risk factors for fractures and falls, including medical history and medication use. In addition, bone mineral density (BMD) measurement with dual-energy X-ray absorptiometry (DXA) of the lumbar spine, total hip, and femoral neck was performed, and a blood sample was collected to detect contributors to secondary osteoporosis and metabolic bone disease [14]. Laboratory tests included serum sodium, potassium, calcium, inorganic phosphate, albumin, creatinine, free tetra-iodothyronine (fT4), thyroidstimulating hormone (TSH), serum aminotransferases (aspartate and alanine amino-transferase), alkaline phosphatase, intact plasma parathyroid hormone (iPTH), serum 25hydroxyvitamin $\mathrm{D}(25(\mathrm{OH}) \mathrm{D})$, and serum protein electrophoresis for all patients. At the FLS, a nurse measured height and weight and evaluated the questionnaire with special attention to medical history, medication use, and calcium intake. Depending on the results of BMD measurements, 25(OH)D levels and calcium intake, patients were treated with calcium supplements, vitamin D supplements, and anti-osteoporosis medication according to the Dutch osteoporosis guideline [8]. Fractures were classified according to Center et al. [15] into hip fractures, major fractures (vertebra, multiple rib, humerus, pelvis, distal femur, and proximal tibia), and minor fractures (all remaining fractures except fingers and toes).

\section{Bone densitometry}

BMD measurements were performed at the lumbar spine (LS; L1-L4), total hip (TH), and femoral neck (FN) using DXA (Hologic QDR 4500, Hologic, Bedford, MA, USA). According to the WHO criteria [16], patients were classified based on the lowest T-score in the LS, TH, and FN. T-scores of $\leq-2.5$ standard deviations (SD) below the reference mean were classified as osteoporosis, T-scores between -1.0 and $-2.5 \mathrm{SD}$ were classified as osteopenia, and T-scores $\geq-1.0$ $\mathrm{SD}$ were classified as normal.

\section{Comorbidities}

Chronic comorbidities in medical history and laboratory tests were classified according to the tenth revision of International Classification of Disease (ICD-10) [17]. In current osteoporosis and fall guidelines [8-10, 18-23], comorbidities with an increased BRC and FRC risk of fractures were identified (Table 1).

\section{Medication use}

Medications were classified according to the Anatomic Therapeutic Chemical (ATC) classification system [24]. In literature [25-28], medication with an increased BRM and FRM risk of fractures were identified (Table 1). Opiates were not included because we could not differentiate between those used chronically and those prescribed related to the recent fracture. Polypharmacy was defined as the use of at least 5 medications at ATC-3 level in which dermatological 
Table 1 Bone- and fall-related comorbidities and medication

Bone-related risk comorbidities (BRC)
Anorexia nervosa
Celiac disease
Chronic kidney disease (CKD)
Chronic obstructive pulmonary disease (COPD)
Diabetes mellitus (DM)
Hemophilia
Hyperthyroidism
Hyperparathyroidism
Hypogonadism
Inflammatory bowel disease (IBD)
Leukemia
Liver cirrhosis
Lymphoma
Malabsorption
Monoclonal gammopathy of unknown significance (MGUS)
Myeloma
Rheumatoid arthritis (RA)
Sarcoidosis
Systemic lupus erythematosus (SLE)
Bone-related risk medication (BRM)
Anticonvulsants
Glucocorticoids, oral
Glucocorticoids, inhaled
H2-receptor inhibitors
Proton pump inhibitors (PPI)
Thiazolidinediones

Fall-related risk comorbidities (FRC)

Arrhythmia

Arthritis

Chronic heart failure

Cognitive impairment

Depression

Diabetes mellitus (DM)

Dizziness

Epilepsy

Incontinence

Osteoarthritis

Parkinson's disease

Peripheral neuropathy

Stroke

Visual impairment
Fall-related risk medications (FRM)
Anti-arrhythmics
Anti-Parkinson medication
Anti-psychotics
Barbiturates
Benzodiazepines
Hypnotics and sedatives
Loop diuretics
Nitrates
Other antidepressants
Selective serotonin reuptake inhibitors (SSRI)
Thiazides
Thiazide-like diuretics
Tricyclic antidepressants (TCA) preparations and medication that was not used chronically were not counted in determining the number of medications.

\section{Statistical analysis}

Results are presented as mean \pm SD or percentages. Data were analyzed using the Chi-square tests and Fisher's exact tests. Subgroup analyses were performed for gender, age per decade, BMD (normal versus osteopenia versus osteoporosis), and fracture type (minor versus major versus hip). Logistic regression analyses were performed to adjust for age, gender, BMD (normal versus osteopenia versus osteoporosis), and fracture type (minor versus major versus hip). All analyses were performed using SPSS for Mac (version 21.0, IBM SPSS Statistics, USA). A $p$ value $\leq 0.05$ was considered as statistically significant.

\section{Results}

From January 2009 until June 2011, 3131 patients aged 50 years or older visited the emergency department with a recent clinical vertebral or non-vertebral fracture. Seventyfour patients were deceased before the invitation for fracture risk evaluation at the FLS was sent, resulting in 3057 patients being invited to the FLS (Fig. 1). Of those, 1694 (55.4\%) patients were willing and able to be evaluated. Included in this study were $1282(41.9 \%)$ FLS patients (71.8\% women and $28.2 \%$ men, mean age $65.0 \pm 9.4$ years) that were fully assessed. Characteristics of these patients are shown in Table 2. Osteoporosis was diagnosed in $30.3 \%$, osteopenia in $47.4 \%$, and $22.3 \%$ had a normal BMD. According to the classification by Center et al. [15], 8.4\% sustained a hip fracture, $30.4 \%$ a major fracture, and $61.2 \%$ a minor fracture. 
Fig. 1 Selection procedure of patients with a fracture

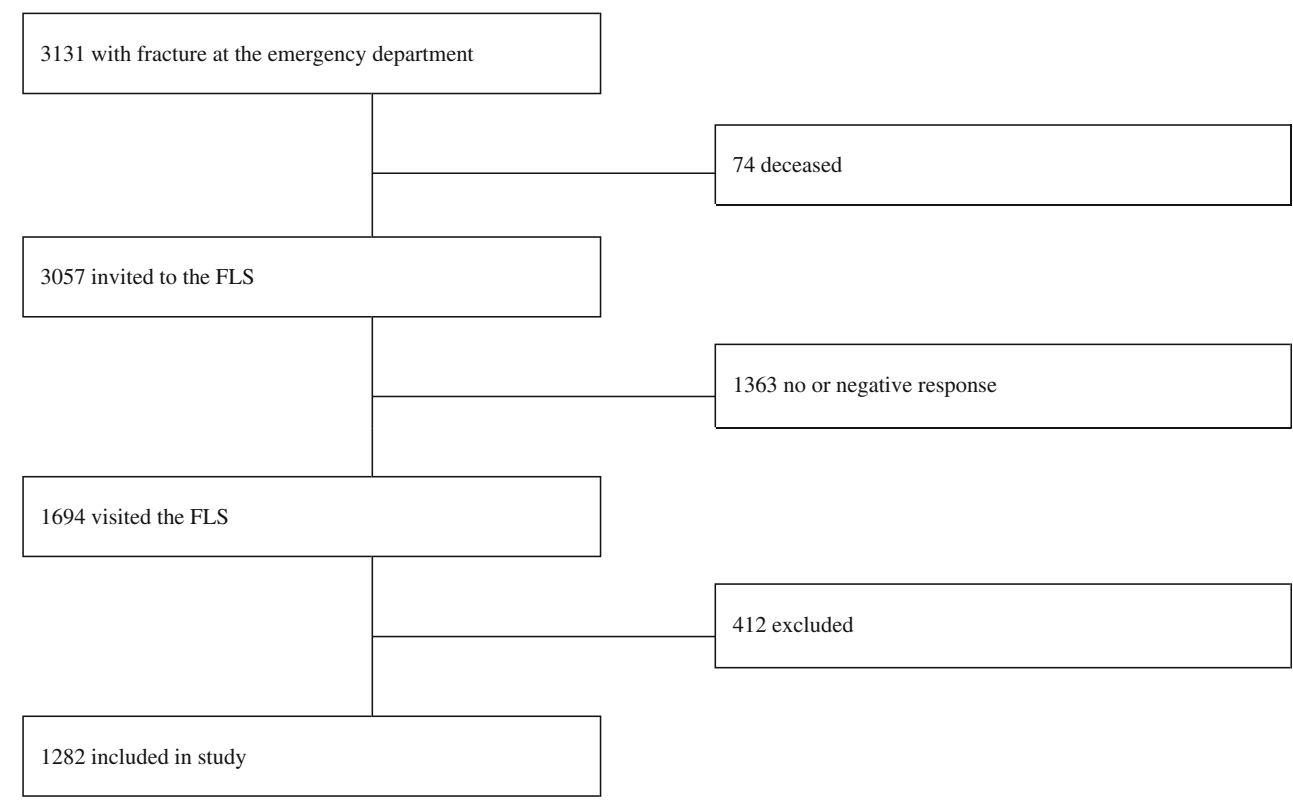

According to BMI, $17 \%$ was obese (i.e., BMI $\geq 30 \mathrm{~kg} / \mathrm{m}^{2}$ ). Previous fractures at 50 + years, previous falls in the last 12 months, and parental history of hip fractures were present in, respectively, $31.0,24.1$, and $1.7 \%$ of patients.

Table 2 Characteristics of the study population

\begin{tabular}{|c|c|c|c|}
\hline & $\begin{array}{l}\text { Total } \\
(n=1282)\end{array}$ & $\begin{array}{l}\text { Men } \\
(n=362)\end{array}$ & $\begin{array}{l}\text { Women } \\
(n=920)\end{array}$ \\
\hline Age, mean \pm SD & $65 \pm 9$ & $64 \pm 9$ & $65 \pm 9$ \\
\hline $50-59$ years & $415(32)$ & $132(37)$ & $283(31)$ \\
\hline $60-69$ years & $446(35)$ & $123(34)$ & $323(35)$ \\
\hline 70-79 years & $307(24)$ & $79(22)$ & $228(25)$ \\
\hline $80+$ years & $114(9)$ & $28(8)$ & $86(9)$ \\
\hline Men & $362(28)$ & & \\
\hline Women & $920(72)$ & & \\
\hline Height, mean \pm SD & $1.67 \pm 0.09$ & $1.76 \pm 0.08$ & $1.64 \pm 0.07$ \\
\hline Weight, mean \pm SD & $73.5 \pm 14.5$ & $82.5 \pm 13.4$ & $70.0 \pm 13.5$ \\
\hline $\mathrm{BMI}$, mean $\pm \mathrm{SD}$ & $26.2 \pm 4.4$ & $26.6 \pm 3.9$ & $16.0 \pm 4.6$ \\
\hline Obesity (BMI $\geq 30 \mathrm{~kg} / \mathrm{m}^{2}$ ) & $193(17.2)$ & $52(16.6)$ & $141(17.5)$ \\
\hline Minor fractures & $784(61)$ & $213(59)$ & $571(62)$ \\
\hline Major fractures & $390(30)$ & $109(30)$ & $281(31)$ \\
\hline Hip fractures & $108(8)$ & $40(11)$ & $68(7)$ \\
\hline Normal BMD & $286(22)$ & $110(30)$ & $176(19)$ \\
\hline Osteopenia & $608(47)$ & $182(50)$ & $426(46)$ \\
\hline Osteoporosis & $388(30)$ & $70(19)$ & $318(5)$ \\
\hline $\begin{array}{l}\text { Previous fractures at } \\
50+\text { years }\end{array}$ & $222(31.0)$ & $50(25.9)$ & $172(33.0)$ \\
\hline Previous falls last 12 months & $252(24.1)$ & $65(21.6)$ & $187(25.1)$ \\
\hline Parental history of hip fractures & $16(1.7)$ & $4(1.3)$ & $12(1.8)$ \\
\hline
\end{tabular}

Data presented as N (\%) unless otherwise stated

\section{Clinical risk factors}

Previous fractures at or above the age of 50 years increased with increasing age (50-59 years: $21.6 \%$ vs. $60-69$ years: $26.2 \%$ vs. $70-79$ years: $40.0 \%$ vs. $80+$ years: $56.9 \%$, $p=.000$ ) and decreasing BMD (normal BMD: $24.6 \%$ vs. osteopenia: $26.4 \%$ vs. osteoporosis $43.1 \%, p=.000$ ). Previous falls in the last 12 months also increased with increasing age (50-59 years: $25.5 \%$ vs. $60-69$ years: $17.9 \%$ vs. $70-79$ years: $24.6 \%$ vs. $80+$ years: $41.1 \%, p=.000)$ and decreasing BMD (normal BMD $22.7 \%$ vs. osteopenia $20.6 \%$ vs. osteoporosis $30.7 \%, p=.004$ ). A parental history of hip fractures was present in $1.7 \%$ of osteoporotic patients, $2.5 \%$ of osteopenic patients, and $0.0 \%$ of those with a normal BMD $(p=.043)$. There were no significant differences in prevalence rates of these risk factors by gender and fracture type.

\section{ICD-10 comorbidities}

As shown in Table 3, $81.0 \%$ of patients had at least one chronic ICD-10 comorbidity, $25.4 \%$ had 1 , and $55.6 \%$ had multiple (up to 13). An overview of the proportion of patients with at least one chronic comorbidity per ICD-10 subgroups is presented in Supplemental Table S1. The prevalence of at least one chronic ICD-10 comorbidity was similar for women and men, and among BMD categories, but increased with increasing BMI (obese: $89.6 \%$ vs. non-obese: $79.3 \%, p=.001$ ), increasing fracture severity (minor fractures: $78.4 \%$ vs. major fractures: $84.6 \%$ vs. hip fractures: $86.1 \%, p=.015$ ) and increasing age ( $72.8 \%$ of patients aged $50-59$ years up to $89.5 \%$ of patients aged $80+$ years, $p<.001$ ) (Table 3 ). In multivariate regression analysis adjusted for age, gender, fracture type and BMD status, age (OR (95\% CI): $1.60(1.35-1.90), p<.001)$, 


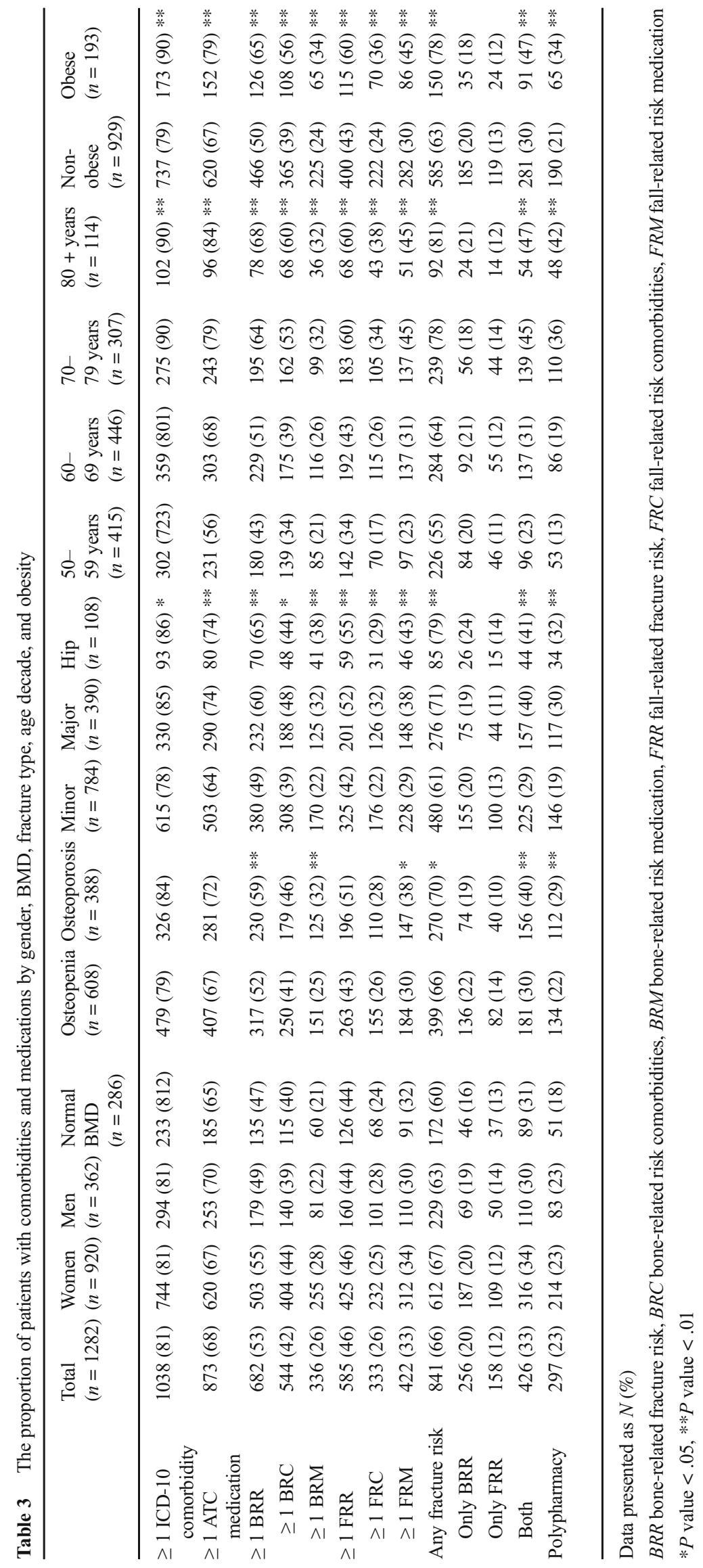


and major fracture (OR (95\% CI): $1.41(1.02-1.96), p=.040)$ were associated with at least one chronic ICD-10 comorbidity. After additional adjustments for BMI, age (1.46 (1.27-1.66), $p<.001)$ and BMI $(1.09(1.05-1.14), p<.001)$ were associated with at least one chronic ICD-10 comorbidity, whereas fracture type was no longer associated.

\section{ATC medication}

The proportion of patients using medication was $68.1 \%$. An overview of the proportion of patients using at least one medication per ATC medication subgroup is presented in Supplemental Table S2. The proportion of patients using at least one medication was similar for women and men, and among BMD categories, but was higher in patients with major and hip fractures compared to those with minor fractures (74.4 vs. 74.1 vs. $64.2 \%$, respectively, $p=.001$ ) and increased with increasing BMI (obese: $78.8 \%$ vs. non-obese: $66.7, p=.001$ ) and increasing age $(55.7 \%$ in patients aged $50-59$ years up to $84.2 \%$ in patients aged $80+$ years, $p<.001$ ) (Table 3 ). In multivariate regression analysis adjusted for age, gender, fracture type, and BMD status, using at least one medication was associated with age (OR (95\% CI) $1.65(1.44-1.90)$ per decade, $p<.001)$ and major fractures (OR $(95 \% \mathrm{CI}) 1.49$ (1.131.97), $p=.004)$. Additional adjustments for BMI showed that in addition to age and fracture severity, BMI (OR (95\% CI): $1.07(1.04-1.11), p<.001)$ was associates with using at least one medical drug.

\section{Comorbidities associated with an increased fracture risk}

At least one comorbidity associated with an increased risk of fractures was found in $50.1 \%$ of patients. At least one bonerelated risk comorbidity (BRC) was found in $42.4 \%$ of patients, with at least one BRC in medical history in $20.2 \%$ and at least one BRC in laboratory tests in $29.4 \%$ of patients. The proportion of patients with at least one BRC in medical history increased significantly with increasing age (5059 years: $16.9 \%$ vs. $60-69$ years: $19.1 \%$ vs. $70-79$ years: $24.4 \%$ vs. $80+$ years: $25.4 \%, p=.036$ ) and increasing BMI (obese: $25.9 \%$ vs. non-obese: $19.1 \%, p=.031$ ). Similarly, the proportion of patients with at least one BRC in laboratory tests increased significantly with increasing age (50-59 years: $20.7 \%$ vs. $60-69$ years: $27.4 \%$ vs. $70-79$ years: $36.5 \%$ vs. $80+$ years: $50.0 \%, p<.001$ ) and increasing BMI (obese: $43 \%$ vs. non-obese: $25.6 \%, p<.001$ ). There were no significant differences in the prevalence rates of at least one BRC in medical history and at least one BRC in laboratory tests between men and women, fracture types, and BMD categories. At least one fall-related risk comorbidity (FRC) was found in $26.0 \%$ of patients (Table 3). Only BRC was present in $24.1 \%$ of patients, only FRC in $7.6 \%$, and a combination of both in $18.3 \%$. A detailed overview of individual BRC and FRC is presented in Supplemental Table S1. Individual BRC in laboratory tests according to age and fractures type are presented in Supplemental Table S3.

\section{Medications associated with increased fracture risk}

At least one medication associated with an increased risk of fractures was used by $44.9 \%$ of patients, with $26.2 \%$ using at least one BRM and $32.9 \%$ at least one FRM (Table 3). Only BRM was used by $11.9 \%$ of patients, only FRM by $18.6 \%$, and a combination of both by $14.3 \%$. A detailed overview of BRM and FRM is presented in Supplemental Table S2.

\section{Bone-related fracture risks}

The proportion of patients with at least one BRC was similar for women and men, and BMD categories, but was significantly higher in obese than in non-obese patients (56 vs. $39 \%, p<.001$ ), in patients with major fractures (48.2\%) and hip fractures (44.4\%) compared to those with minor fractures $(39.3 \%, p=.013)$, and increased with increasing age (33.5\% of patients aged $50-59$ years up to $59.6 \%$ of patients aged $80+$ years, $p<.001$ ) (Table 3 ). In multivariate regression analysis adjusted for age, gender, fracture type, and BMD status, at least one BRC was associated with age (OR $(95 \% \mathrm{CI}): 1.45(1.28-1.64), p<.001)$ and major fractures (OR (95\% CI): $1.36(1.06-1.75)$, $p=.016$ ) (Table 4). Additional adjustments for BMI showed that besides age and fracture severity, BMI (OR (95\% CI): $1.07(1.04-1.10), p<.001)$ was associated with at least one BRC (Supplemental Table S4).

The proportion of patients using at least one BRM were similar for women and men, but increased significantly with increasing BMI (obese: $34 \%$ vs. non-obese: $24 \%, p=.006$ ), decreasing BMD (normal BMD: $21.0 \%$ vs. osteopenia: $24.8 \%$ vs. osteoporosis: $32.2 \%, p=.003)$, increasing fracture severity (minor fractures: $21.7 \%$ vs. major fractures $32.1 \%$ vs. hip fractures: $38.0 \%, p<.001)$, and increasing age $(50-59$ years: $20.5 \%$ vs. $60-69$ years: $26.0 \%$ vs. $70-79$ years: $32.2 \%$ vs. $80+$ years: $31.6 \%, p=.002$ ) (Table 3 ). In multivariate regression analysis adjusted for age, gender, fracture type, and BMD status, age (OR (95\% CI): $1.18(1.03-1.35), p=.019)$, major fractures (OR (95\% CI): $1.63(1.24-2.14), p=.001)$, and hip fractures (OR (95\% CI): $1.98(1.28-3.06) p=.002)$ were associated with at least one BRM (Table 4). After an additional adjustment for BMI, female gender (OR (95\% CI): 1.40 $(1.01-1.94), p=.041)$, increasing BMI (OR $(95 \% \mathrm{CI}): 1.04$ $(1.01-1.07), p=.016)$, and osteoporosis (OR (95\% CI): 1.62 $(1.07-2.45), p=.023)$ were also associated with at least one BRM (Supplemental Table S4).

At least one BRR was present in $53.2 \%$ of patients (only BRC in $27.0 \%$, only BRM in $10.8 \%$, and both in $15.4 \%$ ). The 
Table 4 Univariate and multivariate regression analysis adjusted for gender, age, fracture type and BMD status, for bone- and fall-related risk comorbidities, and medications and their combinations

\begin{tabular}{|c|c|c|c|c|c|c|}
\hline & \multicolumn{2}{|c|}{$\mathrm{BRC}$} & \multicolumn{2}{|c|}{ FRC } & \multicolumn{2}{|c|}{ Any risk comorbidity } \\
\hline & $\begin{array}{l}\text { Univariate } \\
(\mathrm{OR}(95 \% \mathrm{CI}))\end{array}$ & $\begin{array}{l}\text { Multivariate } \\
(\mathrm{OR}(95 \% \mathrm{CI}))\end{array}$ & $\begin{array}{l}\text { Univariate } \\
(\mathrm{OR}(95 \% \mathrm{CI}))\end{array}$ & $\begin{array}{l}\text { Multivariate } \\
(\mathrm{OR}(95 \% \mathrm{CI}))\end{array}$ & $\begin{array}{l}\text { Univariate } \\
(\mathrm{OR}(95 \% \mathrm{CI}))\end{array}$ & $\begin{array}{l}\text { Multivariate } \\
(\mathrm{OR}(95 \% \mathrm{CI}))\end{array}$ \\
\hline Age per decade & $1.47(1.30-1.65) * *$ & $1.45(1.28-1.64) * *$ & $1.49(1.30-1.69) * *$ & $1.47(1.28-1.69) * *$ & $1.56(1.38-1.75) * *$ & $1.52(1.34-1.72) * *$ \\
\hline Male & Reference & Reference & Reference & Reference & Reference & Reference \\
\hline Female & $1.24(0.97-1.59)$ & $1.20(0.93-1.55)$ & $0.87(0.66-1.15)$ & $0.83(0.62-1.10)$ & $1.10(0.86-1.41)$ & $1.04(0.81-1.35)$ \\
\hline Normal BMD & Reference & Reference & Reference & Reference & Reference & Reference \\
\hline Osteopenia & $1.04(0.78-1.38)$ & $0.93(0.70-1.25)$ & $1.10(0.79-1.52)$ & $1.00(0.72-1.41)$ & $1.17(0.88-1.55)$ & $1.05(0.98-1.41)$ \\
\hline Osteoporosis & $1.27(0.94-1.74)$ & $0.97(0.70-1.34)$ & $1.27(0.89-1.80)$ & $1.01(0.70-1.47)$ & $1.45(1.06-1.96) *$ & $1.09(0.79-1.52)$ \\
\hline Minor fracture & Reference & Reference & Reference & Reference & Reference & Reference \\
\hline Major fracture & $1.44(1.13-1.84) * *$ & $1.36(1.06-1.75) *$ & $1.65(1.26-2.16) * *$ & $1.55(1.17-2.04) * *$ & $1.55(1.21-1.98) * *$ & $1.44(1.12-1.85) * *$ \\
\hline \multirow[t]{3}{*}{ Hip fracture } & $1.24(0.82-1.86)$ & $1.05(0.69-1.60)$ & $1.39(0.89-2.18)$ & $1.12(0.70-1.78)$ & $1.41(0.94-2.11)$ & $1.14(0.75-1.73)$ \\
\hline & \multicolumn{2}{|c|}{ BRM } & \multicolumn{2}{|c|}{ FRM } & \multicolumn{2}{|c|}{ Any risk medication } \\
\hline & $\begin{array}{l}\text { Univariate } \\
(\mathrm{OR}(95 \% \mathrm{CI}))\end{array}$ & $\begin{array}{l}\text { Multivariate } \\
(\mathrm{OR}(95 \% \mathrm{CI}))\end{array}$ & $\begin{array}{l}\text { Univariate } \\
(\mathrm{OR}(95 \% \mathrm{CI}))\end{array}$ & $\begin{array}{l}\text { Multivariate } \\
(\mathrm{OR}(95 \% \mathrm{CI}))\end{array}$ & $\begin{array}{l}\text { Univariate } \\
(\mathrm{OR}(95 \% \mathrm{CI}))\end{array}$ & $\begin{array}{l}\text { Multivariate } \\
(\mathrm{OR}(95 \% \mathrm{CI}))\end{array}$ \\
\hline Age per decade & $1.27(1.12-1.45) * *$ & $1.18(1.03-1.35) *$ & $1.49(1.32-1.68) * *$ & $1.44(1.27-1.64) * *$ & $1.51(1.34-1.70) * *$ & $1.43(1.27-1.62) * *$ \\
\hline Male & Reference & Reference & Reference & Reference & Reference & Reference \\
\hline Female & $1.33(0.99-1.77)$ & $1.28(0.96-1.73)$ & $1.18(0.90-1.53)$ & $1.15(0.87-1.50)$ & $1.21(0.95-1.55)$ & $1.17(0.90-1.51)$ \\
\hline Normal BMD & Reference & Reference & Reference & Reference & Reference & Reference \\
\hline Osteopenia & $1.25(0.89-1.75)$ & $1.12(0.79-1.58)$ & $0.93(0.69-1.26)$ & $0.82(0.60-1.12)$ & $1.08(0.82-1.45)$ & $0.96(0.71-1.28)$ \\
\hline Osteoporosis & $1.79(1.26-2.55) * *$ & $1.41(0.97-2.05)$ & $1.31(0.95-1.80)$ & $0.96(0.68-1.36)$ & $1.63(1.20-2.22) * *$ & $1.19(0.86-1.66)$ \\
\hline Minor fracture & Reference & Reference & Reference & Reference & Reference & Reference \\
\hline Major fracture & $1.70(1.30-2.24) * *$ & $1.63(1.24-2.14) * *$ & $1.49(1.15-1.93) * *$ & $1.41(1.09-1.83) *$ & $1.59(1.24-2.02) * *$ & $1.49(1.16-1.91) * *$ \\
\hline \multirow[t]{3}{*}{ Hip fracture } & $2.21(1.45-3.38) * *$ & $1.98(1.28-3.06) * *$ & $1.81(1.20-2.73) * *$ & $1.54(1.00-2.36) *$ & $2.30(1.52-3.47) * *$ & $1.92(1.26-2.94) * *$ \\
\hline & \multicolumn{2}{|l|}{$\mathrm{BRR}$} & \multicolumn{2}{|c|}{ FRR } & \multicolumn{2}{|c|}{ Any risk } \\
\hline & $\begin{array}{l}\text { Univariate } \\
(\mathrm{OR}(95 \% \mathrm{CI}))\end{array}$ & $\begin{array}{l}\text { Multivariate } \\
(\mathrm{OR}(95 \% \mathrm{CI}))\end{array}$ & $\begin{array}{l}\text { Univariate } \\
(\mathrm{OR}(95 \% \mathrm{CI}))\end{array}$ & $\begin{array}{l}\text { Multivariate } \\
(\mathrm{OR}(95 \% \mathrm{CI}))\end{array}$ & $\begin{array}{l}\text { Univariate } \\
(\mathrm{OR}(95 \% \mathrm{CI}))\end{array}$ & $\begin{array}{l}\text { Multivariate } \\
(\mathrm{OR}(95 \% \mathrm{CI}))\end{array}$ \\
\hline Age per decade & $1.46(1.30-1.65) * *$ & $1.39(1.23-1.58) * *$ & $1.54(1.37-1.74) * *$ & $1.50(1.33-1.70) * *$ & $1.62(1.42-1.84) * *$ & $1.56(1.18-3.18) * *$ \\
\hline Male & Reference & Reference & Reference & Reference & Reference & Reference \\
\hline Female & $1.23(0.97-1.57)$ & $1.18(0.92-1.52)$ & $1.08(0.85-1.38)$ & $1.05(0.81-1.35)$ & $1.15(0.90-1.49)$ & $1.12(0.86-1.46)$ \\
\hline Normal BMD & Reference & Reference & Reference & Reference & Reference & Reference \\
\hline Osteopenia & $1.22(0.92-1.62)$ & $1.09(0.82-1.45)$ & $0.97(0.73-1.29)$ & $0.86(0.64-1.15)$ & $1.27(0.95-1.69)$ & $1.12(0.83-1.51)$ \\
\hline Osteoporosis & $1.63(1.20-2.22) * *$ & $1.22(0.88-1.70)$ & $1.30(0.95-1.76)$ & $0.96(0.69-1.34)$ & $1.51(1.10-2.09) *$ & $1.08(0.77-1.52)$ \\
\hline Minor fracture & Reference & Reference & Reference & Reference & Reference & Reference \\
\hline Major fracture & $1.56(1.22-2.00) * *$ & $1.47(1.14-1.88) * *$ & $1.50(1.18-1.92) * *$ & $1.41(1.10-1.81) * *$ & $1.53(1.18-1.99) * *$ & $1.43(1.09-1.86) * *$ \\
\hline Hip fracture & $1.96(1.29-2.98) * *$ & $1.65(1.07-2.54) *$ & $1.70(1.14-2.55) *$ & $1.41(0.93-2.15)$ & $2.34(1.45-3.79) * *$ & $1.93(1.18-3.18) * *$ \\
\hline
\end{tabular}

$B R R$ bone-related fracture risk, $B R C$ bone-related risk comorbidities, $B R M$ bone-related risk medication, $F R R$ fall-related fracture risk, $F R C$ fall-related risk comorbidities, FRM fall-related risk medication

$* P$ value $<.05, * * P$ value $<.01$

proportion of patients with at least one BRR was similar for women and men, but increased significantly with increasing BMI (obese: $65 \%$ vs. non-obese: $50 \%, p<.001$ ), decreasing BMD (normal BMD: $47.2 \%$ vs. osteopenia $52.1 \%$ vs. osteoporosis: $59.3 \%, p=.006$ ), increasing fracture severity (minor fractures $48.5 \%$ vs. major fractures $59.5 \%$ vs. hip fractures $64.8 \%, p<.001)$, and increasing age $(50-59$ years: $43.4 \%$ vs. $60-69$ years: $51.3 \%$ vs. $70-79$ years: $63.5 \%$ vs. $80+$ years: $68.4 \%, p<.001$ ) (Table 3 ). In multivariate analysis adjusted for age, gender, fracture type, and BMD status, age (OR (95\% CI): $1.39(1.23-1.58)$ per decade, $\mathrm{p}<.001)$, major fractures (OR (95\% CI): $1.47(1.14-1.88), p=.003)$, and hip fractures (OR (95\% CI): $1.65(1.07-2.54), p=.023)$ were associated with at least one BRR (Table 4). After additional adjustments for BMI, BMI (OR (95\% CI): $1.06(1.03-1.09), p<.001)$ and osteoporosis (OR (95\% CI): $1.45(1.01-2.08), p=.046)$ were also associated with at least one BRR (Supplemental Table S4). 


\section{Fall-related fracture risks}

The proportion of patients with at least one FRC was also similar for women and men, and BMD categories, but was significantly higher in major fractures (32.3\%) and hip fractures $(28.7 \%)$ compared to those with minor fractures $(22.4 \%, p=.001)$, and increased with increasing BMI (obese: $36 \%$ vs. non-obese: $24 \%, p<.001$ ) and increasing age (16.9\% of patients aged $50-59$ years up to $37.7 \%$ of patients aged $80+$ years, $p<.001$ ) (Table 3 ). In multivariate regression analysis adjusted for age, gender, fracture type, and BMD status, at least one FRC was also associated with age (OR (95\% CI): $1.55(1.17-2.04), p=.002)$ and major fractures (OR (95\% CI): 1.47 (1.28-1.69), $p<.001$ ) (Table 4). Additional adjustments for BMI showed that besides age and fracture severity, BMI (OR (95\% CI): 1.06 (1.02-1.09), $p<.001)$ was associated with at least one FRC (Supplemental Table S4).

The proportion of patients using at least one FRM was also similar for women and men, but was significantly higher in patients with osteoporosis $(37.9 \%)$ compared to those with osteopenia (30.3\%) and a normal BMD (31.8\%, $p=.040)$, and increased with increasing BMI (obese: $45 \%$ vs. nonobese: $30 \%, p<.001$ ), increasing fracture severity (minor fractures: $29.1 \%$ vs. major fractures: $37.9 \%$ vs. hip fractures: $42.6 \%, p=.001)$, and increasing age (50-59 years: $23.4 \%$ vs. 60-69 years: $30.7 \%$ vs. $70-79$ years $44.6 \%$ vs. $80+$ years $44.7 \%, p<.001$ ) (Table 3 ). In multivariate analysis adjusted for age, gender, fracture type, and BMD status, age (OR $(95 \%$ CI): 1.44 (1.27-1.64), $p<.001)$, major fractures (OR $(95 \%$ CI): $1.41(1.09-1.83), p=.010)$, and hip fractures (OR $(95 \%$ CI): $1.54(1.00-2.36), p=.048)$ were associated with at least one FRM (Table 4). Additional adjustments for BMI showed that besides age and fracture severity, BMI (OR (95\% CI): 1.08 (1.04-1.11), $p<.001$ ) was associated with at least one FRM (Supplemental Table S4).

At least one FRR was present in $45.6 \%$ of patients (only FRC in $12.7 \%$, only FRM in $19.7 \%$, and both in $13.3 \%$ ). The proportion of patients with at least one FRR was similar for women and men, and among BMD categories, but increased significantly with increasing fracture severity (minor fractures: $41.5 \%$ vs. major fractures: $51.5 \%$ vs. hip fractures: $54.6 \%, p=.001$ ), increasing BMI (obese: $60 \%$ vs. nonobese $43 \%, p<.001)$, and increasing age (50-59 years: $34.2 \%$ vs. $60-69$ years: $43.0 \%$ vs. $70-79$ years: $59.6 \%$ vs. $80+$ years: $59.6 \%, p<.001$ ) (Table 3). In multivariate analysis adjusted for age, gender, fracture type, and BMD status, age (OR (95\% CI): $1.50(1.33-1.70)$ per decade, $p<.001)$ and major fractures (OR (95\% CI): $1.41(1.10-1.81), p=.001)$ were significantly associated with FRR (Table 4). After additional adjustments for BMI, BMI (OR (95\% CI): 1.08 (1.05$1.11), p<.001)$ was also associated with at least one FRR (Supplemental Table S4).

\section{Any fracture risk}

The proportion of patients having at least one risk (BRC, BRM, FRC, FRM, or any combination) was $65.6 \%$ (only BRR in $20.0 \%$, only FRR in $12.3 \%$, and both in $33.3 \%$ ). The prevalence of at least one risk was similar for women and men, but increased significantly with increasing BMI (obese: $78 \%$ vs. non-obese: $63 \%, p<.001$ ), decreasing BMD (normal BMD: $60.1 \%$ vs. osteopenia: $65.6 \%$ vs. osteoporosis: $69.6 \%, p=.039$ ), with increasing fracture severity (minor fractures: $61.2 \%$ vs. major fractures: $70.8 \%$ vs. hip fractures: $78.7 \%, p<.001)$, and with increasing age $(50$ 59 years: $54.5 \%$ vs. $60-69$ years $63.7 \%$ vs. $70-79$ years $77.9 \%$ vs. $80+$ years: $80.7 \%, p<.001$ ) (Table 3). In multivariate analysis adjusted for age, gender, fracture type and BMD status, age (OR (95\% CI): 1.56 (1.18-3.18) per decade), $p<.001)$, major fracture (OR (95\% CI): 1.43 (1.09-1.86), $p<.001)$, and hip fracture (OR (95\% CI): $1.93(1.18-3.18)$, $p=.009$ ) (Table 4). Additional adjustment for BMI showed that besides age and fracture severity, BMI (OR (95\% CI): $1.08(1.05-1.12), p<.001)$ was associated with at least one risk (Supplemental Table S4).

As shown in Table 3, the proportion of patients with only BRR as well as the proportion of patients with only FRR were similar among gender, BMI, BMD, fracture, and age subgroups. In contrast, the proportion of patients with a combination of BRR and FRR was similar for women and men, but significantly higher in obese compared to non-obese patients (47 vs. $30 \%, p<.001)$, in patients with osteoporosis $(40.2 \%)$ compared to those with osteopenia $(29.8 \%)$ and a normal BMD $(31.1 \%, p=.002)$, higher in patients with major fractures $(40.3 \%)$ and hip fractures $(40.7 \%)$ compared to minor fractures $(28.7 \%, p<.001)$, and increased significantly with increasing age per decade (50-59 years: $23.1 \%$ vs. 60 69 years: $30.7 \%$ vs. $70-79$ years: $45.3 \%$ vs. 80 + years: $47.4 \%, p<.001$ ) (Fig. 2). In multivariate analysis adjusted for age, gender, fracture type, and BMD status, the combination of BRR and FRR was significantly associated with age per decade (OR (95\% CI): 1.47 (1.30-1.68), $p<.001)$ and major fracture (OR (95\% CI): $1.58(1.21-2.04), p=.001)$. Additional adjustments for BMI showed that besides age and fracture severity, BMI (OR (95\% CI): 1.08 (1.05-1.12), $p<.001)$ was associated with at least one risk.

\section{Polypharmacy}

Polypharmacy was present in $23.2 \%$ of patients (Table 3 ). The prevalence of polypharmacy was similar for women and men, and all fracture locations, but was significantly higher in patients with osteoporosis compared to those with osteopenia, and a normal BMD (28.9 vs. 22.0 vs. $17.8 \%, p=.002$ ) (Table 2), and increased with increasing BMI (obese $33.7 \%$ vs. $20.5 \%, p<.001)$ and increasing age from $12.8 \%$ in 


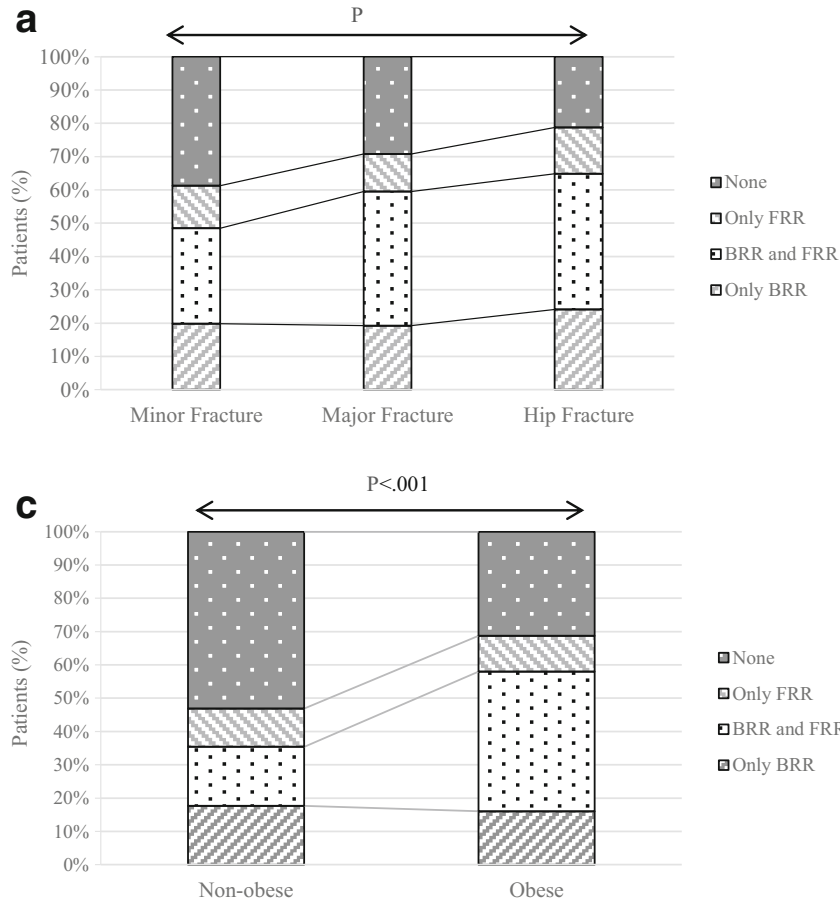

Fig. 2 Proportion of patients with only bone-related fracture risks, only fall-related fracture risks, a combination of both, and none according to fracture type (a), age per decade (b), and obesity (c). The proportion of patients with only BRR $( \pm 20 \%)$ and the proprotion of patients with only

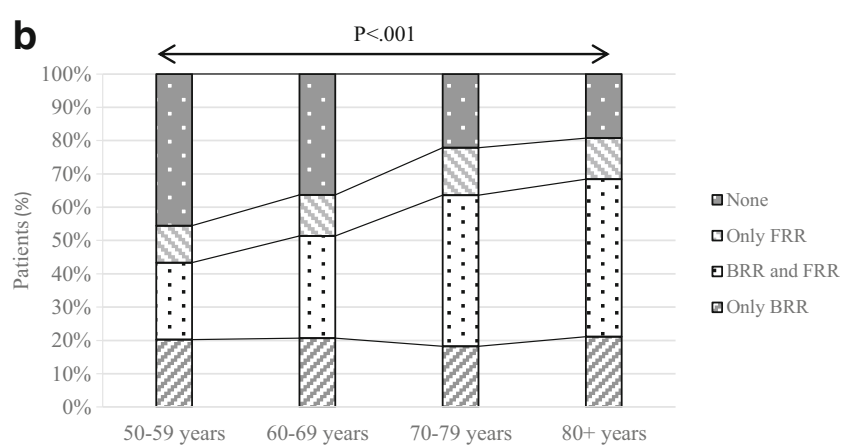

FRR $( \pm 10 \%)$ remained constant; whereas, the proportion of patients with a combination of BRR and FRR increased significantly with fracture severity $(p<.001)$, increasing age $(p<.001)$, and obesity $(p<.001)$. $B R R$ bone-related fracture risk, $F R R$ fall-related fracture risk

gender, age, BMI, BMD, and fracture type subgroups, whereas the proportion of patients having a combination of BRR and FRR increased significantly with increasing age, BMI, and severity of the fracture. These findings imply that comorbidities and medications associated with a bone- or fall-related risk of fractures are often present in FLS patients and that bone- and fall-related fracture risk often co-exist, especially in patients at older age, higher BMI, and with more severe fractures.

Several but not all fracture risk calculators include comorbidities and medications in their models. They are implemented separately in the QFracture risk calculator [29], indicating that the risk for fractures increases with the number of mentioned comorbidities and medications. Apart from rheumatoid arthritis and glucocorticoid use, other comorbidities and medications are included as a combined risk factor (secondary osteoporosis, regardless of the number of comorbidities), not taking into account the number and severity, in the fracture risk assessment tool (FRAX) [30]. Comorbidities and medications are not included in the Garvan fracture risk calculator $[31,32]$. One study [33] investigated the number of comorbidities in relation to subsequent fracture risk and reported hazard ratio of 2.0 for subsequent fracture over 7 years in the presence of $>3$ comorbidities, independent of the use of glucocorticoids (hazard ratio 1.75). Therefore, documenting the comorbidities and medications in patients attending the having at least one BRR or at least one FRR was similar for 
FLS contributes to a more profound assessment of subsequent fracture risk, but more prospective studies will be needed to evaluate the additive or synergistic effects of multiple risk factors on fracture risk.

This study provides a detailed overview of comorbidities and medications in patients able and willing to visit the FLS, but these findings may not be generalized to all other patients with a recent fracture. In this study, $42 \%$ of all invited patients with a recent fracture attended the FLS. From previous studies, we know that patients who were not willing or able to have their fracture risk evaluated at the FLS were older and more frequently had a hip fracture [34-37]. Consequently, in the non-attenders, the proportion of patients with BRR and FRR may be even higher.

In conclusion, comorbidities and medications associated with an increased bone- or fall-related fracture risk are present in two-thirds of patients attending the FLS after a recent fracture. Additionally, the proportion of patients only having at least one BRR or at least one FRR was similar for gender, age, BMI, BMD, and fracture type subgroups, whereas the proportion of patients having a combination of BRR and FRR increased significantly with increasing age, BMI, and severity of the fracture. This indicates that systematic evaluation of these factors is important for a more profound assessment of subsequent fracture risk in FLS care.

Funding This study was supported by funding of the Weijerhorst Foundation.

\section{Compliance with ethical standards}

Conflicts of interest Dr. Vranken declares that she has no conflict of interest.

Dr. Wyers declares that she has no conflict of interest.

Dr. Van der Velde declares that he has no conflict of interest.

Dr. Janzing declares that he has no conflict of interest.

Dr. Kaarsemaker declares that he has no conflict of interest.

Dr. Geusens reports grants and other from Amgen, grants from Pfizer, grants from MSD, grants from UCB, grants from Abbott, grants and other from Lilly, grants from BMS, grants from Novartis, grants from Roche, and grants from Will Pharma, outside the submitted work.

Dr. Van den Bergh reports grants from Amgen, grants from Will Pharma, and grants from Eli Lilly, outside the submitted work.

Open Access This article is distributed under the terms of the Creative Commons Attribution-NonCommercial 4.0 International License (http:// creativecommons.org/licenses/by-nc/4.0/), which permits any noncommercial use, distribution, and reproduction in any medium, provided you give appropriate credit to the original author(s) and the source, provide a link to the Creative Commons license, and indicate if changes were made.

\section{References}

1. Kanis JA, Johnell O, Oden A et al (2000) Long-term risk of osteoporotic fracture in Malmö. Osteoporos Int 11:669-674. https://doi. org/10.1007/s001980070064
2. van Staa TP, Dennison EM, Leufkens HGM, Cooper C (2001) Epidemiology of fractures in England and Wales. Bone 29:517522. https://doi.org/10.1016/S8756-3282(01)00614-7

3. Hernlund E, Svedbom A, Ivergård M et al (2013) Osteoporosis in the European Union: medical management, epidemiology and economic burden. Arch Osteoporos 8:136. https://doi.org/10.1007/ s11657-013-0136-1

4. Kanis JA, Johnell O, De Laet C et al (2004) A meta-analysis of previous fracture and subsequent fracture risk. Bone 35:375-382. https://doi.org/10.1016/j.bone.2004.03.024

5. Center JR, Bliuc D, Nguyen TV, Eisman JA (2007) Risk of subsequent fracture after low-trauma fracture in men and women. JAMA 297:387-394. https://doi.org/10.1001/jama.297.4.387

6. Klotzbuecher CM, Ross PD, Landsman PB et al (2010) Patients with prior fractures have an increased risk of future fractures: a summary of the literature and statistical synthesis. J Bone Miner Res 15:721-739. https://doi.org/10.1359/jbmr.2000.15.4.721

7. van Geel AC, Geusens PP, Nagtzaam IF et al (2006) Timing and risk factors for clinical fractures among postmenopausal women: a 5-year prospective study. BMC Med 4:1-7. https://doi.org/10.1186/ 1741-7015-4-24

8. Dutch Institute for Healthcare Improvement CBO (2011) Richtlijn Osteoporose en Fractuurpreventie, Derde Herziening [Dutch] Utrecht, Dutch Institute for Healthcare Improvement CBO, The Netherlands

9. Compston J, Bowring C, Cooper A et al (2013) Diagnosis and management of osteoporosis in postmenopausal women and older men in the UK: National Osteoporosis Guideline Group (NOGG) update 2013. Maturitas 75:392-396. https://doi.org/10.1016/j. maturitas.2013.05.013

10. Cosman F, de Beur SJ, LeBoff MS et al (2014) Clinician's guide to prevention and treatment of osteoporosis. Osteoporos Int 25:2359 2381. https://doi.org/10.1007/s00198-014-2794-2

11. Eisman JA, Bogoch ER, Dell R et al (2012) Making the first fracture the last fracture: ASBMR task force report on secondary fracture prevention. J Bone Miner Res 27:2039-2046. https://doi.org/ 10.1002/jbmr. 1698

12. Lems WF, Dreinhöfer KE, Bischoff-Ferrari H et al (2016) EULAR/ EFFORT recommendations for management of patients older than 50 years with a fragility fracture and prevention of subsequent fractures. Ann Rheum Dis. https://doi.org/10.1136/annrheumdis-2016210289

13. IOF Fracture Working Group, Åkesson K, Marsh D et al (2013) Capture the fracture: a best practice framework and global campaign to break the fragility fracture cycle. Osteoporos Int 24: 2135-2152. https://doi.org/10.1007/s00198-013-2348-z

14. Bours SPG, van Geel TACM, Geusens PPMM et al (2011) Contributors to secondary osteoporosis and metabolic bone diseases in patients presenting with a clinical fracture. J Clin Endocrinol Metab 96:1360-1367. https://doi.org/10.1210/jc.2010-2135

15. Center JR, Nguyen TV, Schneider D et al (1999) Mortality after all major types of osteoporotic fracture in men and women: an observational study. The Lancet 353:878-882. https://doi.org/10.1016/ S0140-6736(98)09075-8

16. World Health Organisation (2003) Prevention and management of osteoporosis, World Health Organization Technical Report Series, vol. 921, pp. 1-164

17. World Health Organization WHO international statistical classification of diseases and related health problems $10^{\text {th }}$ revision

18. IOF CSA Fracture Working Group, Marsh D, Åkesson K et al (2011) Coordinator-based systems for secondary prevention in fragility fracture patients. Osteoporos Int 22:2051-2065. https://doi. org/10.1007/s00198-011-1642-x

19. World Health Organization (2007) WHO global report on falls prevention in older age. 
20. Dutch Institute for Healthcare Improvement CBO (2004) Richtlijn Preventie van valincidenten bij ouderen [Dutch] Utrecht, Dutch Institute for Healthcare Improvement CBO, The Netherlands

21. National Institute for Health and Care Excellence (2015) Falls in older people.

22. Panel on falls prevention, American Geriatrics Society, British Geriatrics Society, and American Academy of Orthopaedic Surgeons (2001) Guideline for the prevention of falls in older persons. J Am Geriatr Soc. 49(5):664-72

23. Panel on Prevention of Falls in Older Persons, American Geriatrics Society and British Geriatrics Society (2011) Summary of the updated American Geriatrics Society/British Geriatrics Society clinical practice guideline for prevention of falls in older persons. J Am Geriatr Soc 59:148-157. https://doi.org/10.1111/j.1532-5415.2010. 03234.x

24. WHO Collaborating Centre for Drug Statistics Methodology (2016) Guideline for ATC classification and DDD assignment, 20th edition

25. Sjöberg C, Bladh L, Klintberg L et al (2010) Treatment with fallrisk-increasing and fracture-preventing drugs before and after a hip fracture: an observational study. Drugs Aging 27:653-661. https:// doi.org/10.2165/11538200-000000000-00000

26. Woolcott JC, Richardson KJ, Wiens MO et al (2009) Meta-analysis of the impact of 9 medication classes on falls in elderly persons. Arch Intern Med 169:1952-1960

27. Ham AC, Swart KMA, Enneman AW et al (2014) Medicationrelated fall incidents in an older, ambulant population: the BPROOF study. Drugs Aging 31:917-927. https://doi.org/10.1007/ s40266-014-0225-x

28. Munson JC, Bynum JPW, Bell J-E et al (2016) Patterns of prescription drug use before and after fragility fracture. JAMA Intern Med 176:1531-1538. https://doi.org/10.1001/jamainternmed.2016.4814

29. Hippisley-Cox J, Coupland C (2009) Predicting risk of osteoporotic fracture in men and women in England and Wales: prospective derivation and validation of QFractureScores. BMJ 339:b4229b4229. https://doi.org/10.1136/bmj.b4229

30. Kanis JA, Oden A, Johnell O et al (2007) The use of clinical risk factors enhances the performance of BMD in the prediction of hip and osteoporotic fractures in men and women. Osteoporos Int 18: 1033-1046. https://doi.org/10.1007/s00198-007-0343-y

31. Nguyen ND, Frost SA, Center JR et al (2007) Development of a nomogram for individualizing hip fracture risk in men and women. Osteoporos Int 18:1109-1117. https://doi.org/10.1007/s00198-0070362-8

32. Nguyen ND, Frost SA, Center JR et al (2008) Development of prognostic nomograms for individualizing 5-year and 10-year fracture risks. Osteoporos Int 19:1431-1444. https://doi.org/10.1007/ s00198-008-0588-0

33. Ganda K, Schaffer A, Seibel MJ (2014) Predictors of re-fracture amongst patients managed within a secondary fracture prevention program: a 7-year prospective study. Osteoporos Int 26:543-551. https://doi.org/10.1007/s00198-014-2880-5

34. Eekman DA, van Helden SH, Huisman AM et al (2013) Optimizing fracture prevention: the fracture liaison service, an observational study. Osteoporos Int 25:701-709. https://doi.org/10.1007/ s00198-013-2481-8

35. Malgo F, Appelman-Dijkstra NM, Termaat MF et al (2016) High prevalence of secondary factors for bone fragility in patients with a recent fracture independently of BMD. Arch Osteoporos 11:12. https://doi.org/10.1007/s11657-016-0258-3

36. van Helden S, van Geel AC, Geusens PP et al (2008) Bone and fallrelated fracture risks in women and men with a recent clinical fracture. J Bone Joint Surg Am Vol 90:241-248. https://doi.org/10. 2106/JBJS.G.00150

37. Naranjo A, Ojeda-Bruno S, Bilbao Cantarero A et al (2014) Results of a model of secondary prevention for osteoporotic fracture coordinated by rheumatology and focused on the nurse and primary care physicians. Reumatol Clín (English Edition) 10:299-303. https:// doi.org/10.1016/j.reumae.2014.03.004 